\title{
Tracking Method of Adaptive For Inserting an Artificial Lens
}

\author{
Kyung-Pyo Kim, Jae-Hyun Jun, Dong-Sik Park, Dong-Jun Lee, and Sung-Ho Kim
}

\begin{abstract}
We marked with a pen directly to the incision for insertion into the patient's eye of the intraocular lens insertion in cataract surgery, and insertion point. However, it can delete or blur the display due to such factors as foreign material coming out of the eye during surgery. Therefore, in recent years, a method of tracking the image processing by receiving the camera images in real time display method such extended. There are many ways to estimate the object in the video in, but in the method has been applied widely used typically have optical flow and template matching. The template matching, while indicating the tracking performance with excellent background simple from complex background, its performance is reduced, while indicating the tracking excellent performance in a complex background, simple background, optical flow, the performance fall. In this paper, there is provided a method adaptive tracking don't give a significant effect on the change in the background template region. Therefore, by using to suit the circumstances the optical flow indicating the tracking excellent performance in a complex background template matching showing a tracking superior performance with a simple background, when inserting an intraocular lens in cataract surgery, and a knife in it was possible to improve the accuracy of tracking a region of the incision.
\end{abstract}

Index Terms-Adaptive tracking method, optical flow, correlation coefficient, template matching.

\section{INTRODUCTION}

Nowadays cataract surgery is one of the most frequent surgeries in Korean society. This cataract appears mainly from adult over the age of 50 mainly, frequency of occurrence because he even higher due to the aging of our society today. Just need a lot of cataract surgery, the treatment also varies, the method most widely used, is a surgical procedure that uses an artificial lens.

The surgery can be inserted exactly according to the angle and points that are calculated in advance in the intraocular lens insertion time is important, before inserting the intraocular lens in cataract surgery when the current mark with a pen directly on the patient's eye to, and shows a lens angle which is inserted incision for insertion.

For this reason, display or blurred due to foreign matter and other secretions that point you have marked with a pen in advance come out to the eye during surgery, in the worst case, it can display itself is deleted. So, accurate tracking of the insertion region is very important to the artificial lens.

The object tracking technology is many methods. Optical Flow, Template Matching, many ways such as SURF(Speeded Up Robust Features), SIFT(Scale Invariant

Manuscript received June 22, 2014; revised August 23, 2014.

The authors are with School of Computer Science and Engineering, Kyungpook National University, Daegu, Republic of Korea (e-mail: kpkim@mmlab.knu.ac.kr; kpkim@mmlab.knu.ac.kr).
Feature Transform), KLT(Kanade Lucas Tomasi Feature Tracker) has been investigated [1], [2].

As mentioned earlier, the biggest advantage of the template matching is easy. Tracking performance rather than to decline to say whether, in the case of background template region is not complicated, and show superior performance. However, the background of the insertion space changes the artificial lens. You can be simple or complex depending on the status of the surgery. Therefore, in this paper, there is provided a method for adaptive tracking using template matching and optical flow for accurate tracking of the insertion region an artificial lens.

The paper is organized as follows. Section II describes optical flow used in this paper, a method of template matching, in Section III, you will learn how to adaptive tracking proposed. Then, in Section IV, and the results and analysis through direct experiment of the proposed system, we present the conclusions in Section $\mathrm{V}$ at the finish.

\section{The Tracking Method OF INSERTION REGION AN ARTIFICIAL LENS}

\section{A. Optical Flow}

Optical flow refers to a pattern of an object motion caused by relative motion between the observation target and (cameras and eyes) observer in the image surface of the edge. Then, it may be utilized flow of light generated within the image, or split tracking a region of interest.

A method of tracking the pattern of the luminance values between frames of a video continuous optical flow is known as the most useful method which can track the change in brightness in space time [3].

The method is divided into rare optical flow and dense optical flow. Since the complexity of the change in velocity and displacement of all pixels that are present in the image is very much, you do not have track the movement of large objects, dense optical flow, and to implement in real time have a difficult. On the other hand, to be used to have only a small area basically, it is possible to calculate the amount appears very small compared to the dense optical flow to track the movement of large objects, rare optical flow implementation in real-time it is possible. Therefore, in this paper, I have applied the Lucas-Kanade algorithm that is used most widely in the way of rare optical flow that can be implemented in real time [3], [4].

Based on the assumption of brightness enhancement (Brightness constancy), temporal persistence, three integrity of space (spatial coherence), Lucas-Kanade algorithm, to track the feature region. Pixel What on the object, on the assumption that even if the frame changes, the value does not change, time duration is assumed to move in the image 
and not fast so much improvement of the brightness. That is, the time changes are made more quickly than the movement of objects in the image, which means that the amount of movement of objects between consecutive frames is not many. Finally, the consistency of the space, it is assumed likely to belong to the same object has high points are adjacent to each other in space, and have the same motion. An explanation of denoted as Brightness values $I$, pixel location $x$, time $t$.

The improvement in brightness, over time pixels in a certain area being tracked shows that the constant is the value. This can be expressed by the formula as follows.

$$
f(x, t) \equiv I(x(t), t)=I(x(t+d t), t+d t)
$$

Equation (1), applying an additional velocity component in the $x$ and $y$ axes of the chain rule and the partial derivatives, it can be expressed as follows.

$$
\begin{gathered}
I_{x_{1}} V_{x}+I_{y_{1}} V_{y}=-I_{t_{1}} \\
I_{x_{2}} V_{x}+I_{y_{2}} V_{y}=-I_{t_{2}} \\
\vdots \\
I_{x_{n}} V_{x}+I_{y_{n}} V_{y}=-I_{t_{n}}
\end{gathered}
$$

$V_{x}$ and $V_{y}$ of equation (2) represents the velocity component of each axis, I represents the intensity of the brightness of each pixel. It is possible to transform equation (3) based on the assumption of space consistent equation (2)

$$
\left[\begin{array}{cc}
I_{x_{1}} & I_{y_{1}} \\
I_{x_{2}} & I_{y_{2}} \\
\vdots & \vdots \\
I_{y_{1}} & I_{y_{1}}
\end{array}\right]\left[\begin{array}{l}
V_{x} \\
V_{y}
\end{array}\right]=\left[\begin{array}{c}
-I_{t_{1}} \\
-I_{t_{2}} \\
\vdots \\
-I_{t_{n}}
\end{array}\right]
$$

In Over constrained system capable of interpretation from a single edge, the formula (3) may be derived in the form of equation (4) by applying the least squares method.

$$
\left[\begin{array}{lr}
\sum I_{x_{i}}^{2} & \sum I_{x_{i}} I_{y_{i}} \\
\sum I_{x_{i}} I_{y_{i}} & \sum I_{y_{i}}^{2}
\end{array}\right]\left[\begin{array}{l}
V_{x} \\
V_{y}
\end{array}\right]=-\left[\begin{array}{l}
\sum I_{x_{i}} I_{t_{i}} \\
\sum I_{y_{i}} I_{t_{i}}
\end{array}\right]
$$

It is possible to derive $V_{x}$ and $V_{y}$ showing the axial velocity component from the above equation.

Brightness-enhancing, duration, Lucas-Kanade algorithm to track a specific area within the image based on three assumptions space consistency as described above.

\section{B. Template Matching}

Template matching is a method that performs matching by using the correlation between the input images created as a template image of a region to try tracking. Template matching, there have three ways. It is squared difference method, correlation matching method and correlation coefficient method. $I$ an input image, $T$ template, referred to as $R$ a picture of the results for that explanation [5], [6].

Squared difference matching method uses the car of the squares of the pixel values. Therefore, now returns 0 if an exact match, otherwise, the value is increased. This can be expressed by the formula as follows.

$$
R_{s q_{d i f f}}(x, y)=\sum_{x^{\prime}, y^{\prime}}\left[T\left(x^{\prime}, y^{\prime}\right)-I\left(x+x^{\prime}, y+y^{\prime}\right)\right]^{2}
$$

The correlation matching method, add all the squares to the product of the input image and the template.

Therefore, the value of exact match is out big, value of the case does not match or out, 0 comes out. This can be expressed by the formula as follows.

$$
R_{c c o r r}(x, y)=\sum_{x^{\prime}, y^{\prime}}\left[T\left(x^{\prime}, y^{\prime}\right) \cdot I\left(x+x^{\prime}, y+y^{\prime}\right)\right]^{2}
$$

Method of correlation coefficient, executes the matching considering the average of the input image and the template. Therefore, returns -1 if it returns 1 matches perfectly, perfectly matched, it means that there is no relationship at all between both images. This can be expressed by the formula as follows.

$$
\begin{gathered}
R_{\text {ccoeff }}(x, y)=\sum_{x^{\prime}, y^{\prime}}\left[T\left(x^{\prime}, y^{\prime}\right) \cdot I\left(x+x^{\prime}, y+y^{\prime}\right)\right]^{2} \\
T^{\prime}\left(x^{\prime}, y^{\prime}\right)=T\left(x^{\prime}, y^{\prime}\right)-\frac{\sum_{x^{\prime \prime}, y^{\prime \prime}} T\left(x^{\prime \prime}, y^{\prime \prime}\right)}{w \cdot h} \\
I^{\prime}\left(x+x^{\prime}, y+y^{\prime}\right)= \\
I^{\prime}\left(x+x^{\prime}, y+y^{\prime}\right)-\frac{\sum_{x^{\prime \prime}, y^{\prime \prime}} T\left(x+x^{\prime \prime}, y+y^{\prime \prime}\right)}{w \cdot h}
\end{gathered}
$$

$h$ and $w$ represents the width and height of the template region, respectively. By calculating a correlation coefficient using each average of the input image and the template region, and not in the following manner squares simple generally better to use a matching method of a complex shape, such as the correlation coefficient is more it is possible to obtain accurate results.

\section{THE Proposed METHOD}

As described above, if the background of the template region is simple, template matching, show excellent performance, but if the background is complex, it is possible to track by mistake. However, the optical flow, for recording by using the feature points in the template region, the background of the template region shows superior performance for complex cases. In this paper, there is provided a method for adaptive tracking was applied only advantages of both methods. Structure of the proposed method is as follow as Fig. 1.

First, you must determine whether the complex background is a simple background change is not large background of the template area to track. This is possible without calculating separately, it is determined by using the correlation coefficient is determined by template matching.

This is noted that indicates the performance template matching excellent simple background, tracking performance of complex background is reduced. After all, I can be the value of the correlation coefficient to the decision to a simple background closer to 1 .

Get the most appropriate threshold value $\mathrm{x}$ through the simulation, if the value of the correlation coefficient is greater than the threshold value $\mathrm{x}$, and as long as they have the same or less or background, simple, we determined the complex background, it is possible to apply each of the 
template matching and optical flow according to the situation.

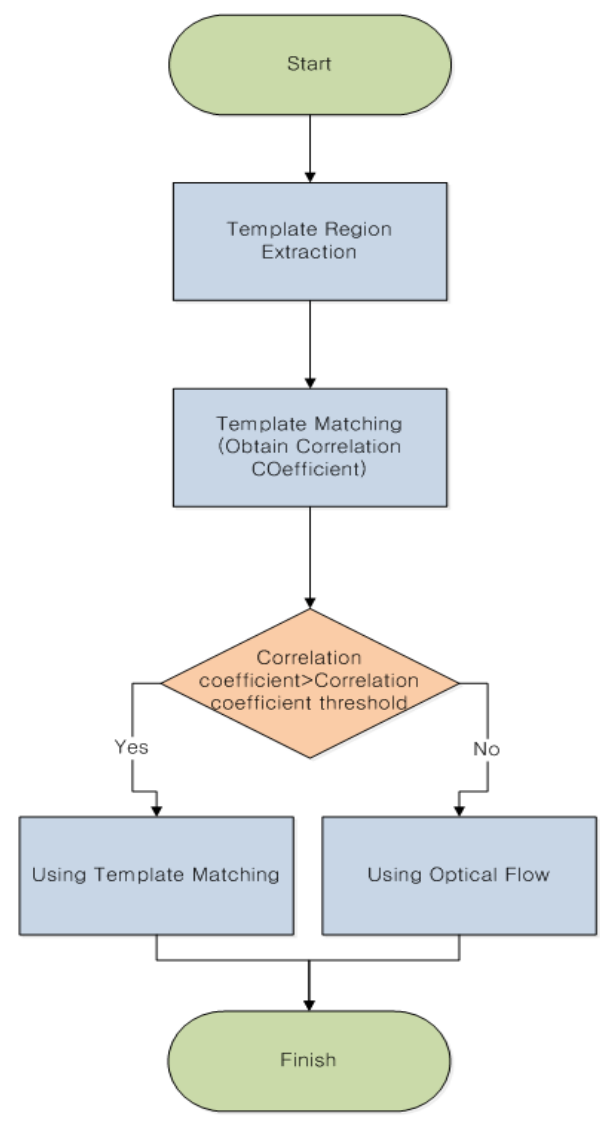

Fig. 1. Structure of the adaptive tracking method.

\section{EXPERIMENTAL RESULTS AND ANALYSIS}

Applying each of the proposed method with template matching with respect to the frame, such as a test image for evaluating the performance of the proposed method, we compared the performance of a particular region.

\section{A. Experiment Environment}

First, we used Visual Studio 2010 for Microsoft Soft, Inc. as a development tool for experimentation. It has been widely used for image processing smoothly, using a computer vision library OpenCV2.2 being developed by the Open Source.

In order to apply for the same frame the way of each trace, and if necessary to compare, experimental video, using surgery video where you want to insert the artificial lens in fact, this is to capture and process surgical video in real time no problem even when viewed in the process.

Prior to experiments the proposed method, an experiment was performed on the image various materials in order to obtain the threshold value $\mathrm{x}$ of the correlation coefficient. This is an experiment for determining the value of the correlation coefficient in the case of the wrong track when you specify a template region specific for each video article attempts to template matching.

Result of the experiment, there was a difference little by little each time for each movie the maximum correlation coefficient in the case of incorrect tracking for visual materials. However, it is determined that the threshold value of the correlation coefficient of the best when it is applied to the proposed algorithm the threshold 0.9 to fine, and the difference was used as the threshold value of the correlation coefficient of 0.9 .

\section{B. Results and Discussion}

The following is a picture when applying the method described above as when using a $0.9 \times$ threshold of the correlation coefficient, template matching is applied to the same frame.

Fig. 2 were respectively applied the proposed method with template matching for surgical recorded video of the actual frame is fps 29 width 720 and height 480 . Then, it is obtained by capturing video screen when the frame 1100 (b, d) in the case of the 0.99 frame. At $150(a, c)$ frames, specify the template any point, we capture a frame trace results were different. (b), (d) is a screen shot of image after seeded saline pupil of the patient during surgery. As shown in the figure, the (b), the (d), and changes in background template region case of applying the method can be seen that the trace is wrong, we have proposed the case of applying a template matching it was confirmed that the track is exactly also big thing that's changed.

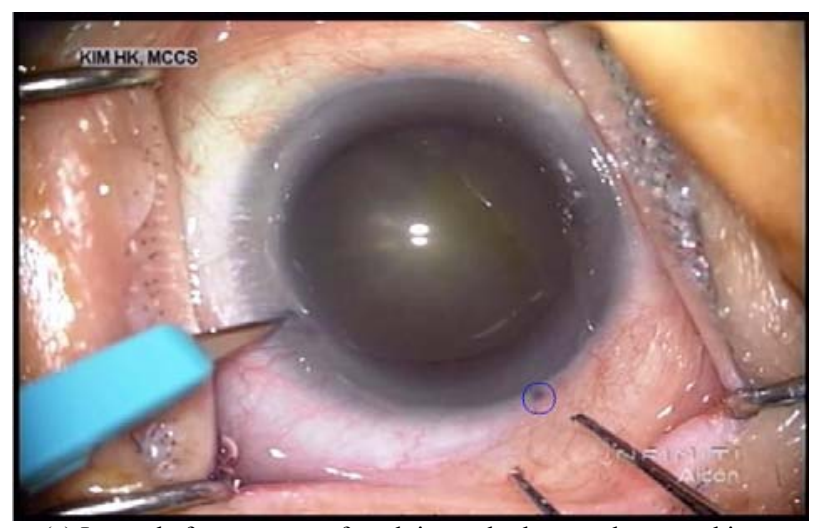

(a) Image before surgery of applying only the template matching.

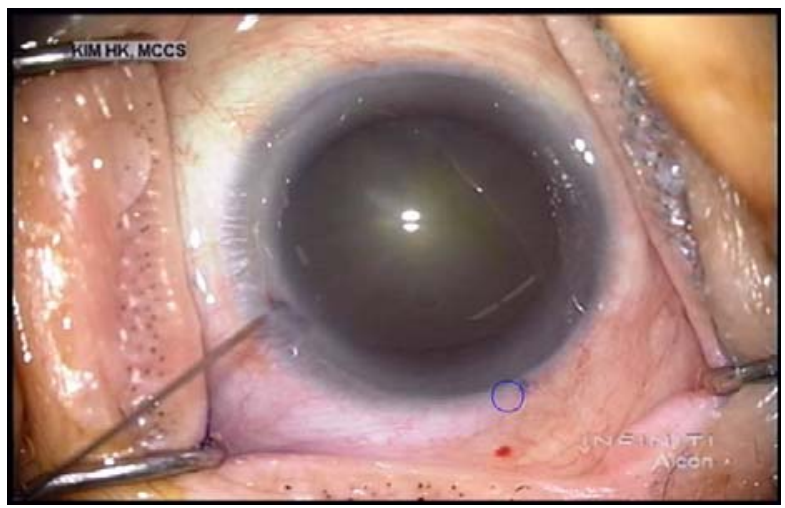

(b) Image during surgery of applying only template matching.

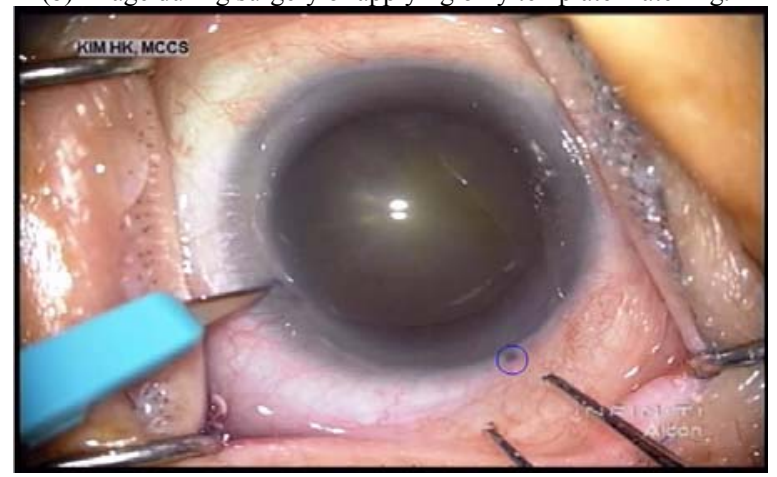

(c) Image before surgery of applying the proposed method. 


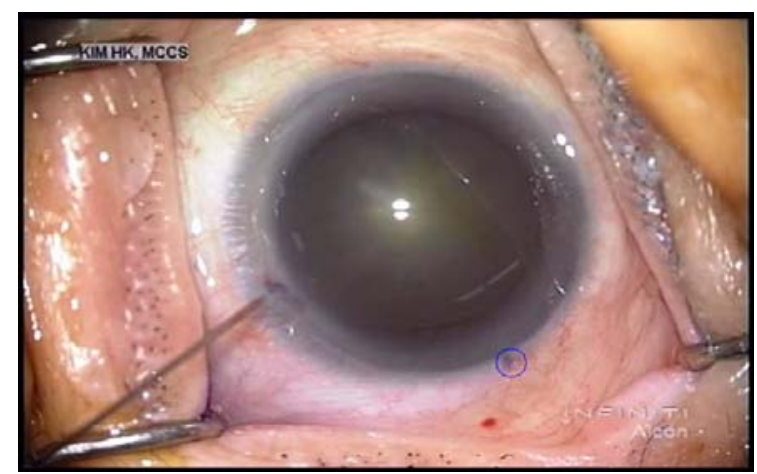

(d) During surgery image of applying the proposed method.

Fig. 2. Results of applying the proposed method and results the case of applying a template matching on the experimental image.

\section{CONCLUSION}

In this paper, we proposed a method of adaptive additional to using the template matching and optical flow, applied only advantages of both methods. It is possible to apply the method by obtaining the threshold $\mathrm{x}$ of the correlation coefficient that best through simulation, to determine the background template region and tracked in accordance with the situation. Thus, we can, regardless of the changed of the background template region, see tracking performance.

However, we need to raise the question about what to determine only the correlation coefficient changes in the background of the template area. Of course, in the experiment of the materials collected, the problem did not occur, there is data that can be a result of an exception is certainly see. Therefore, studies to find a reference to determine the exact change in the background of the template region is required.

\section{REFERENCES}

[1] M. Bicego, A. Lagorio, E. Grosso, and M. Tistarelli, "On the use of SIFT features for face authentication," in Proc. the Conference on Computer Vision and Pattern Recognition Workshop (CVPRW'06), 2006, pp. 35.

[2] M. Hwangbo, J. S. Kim, and T. Kanade, "Inertial-Aided KLT feature tracking for a moving camera," in Proc. the Conference on Intelligent Robots and Systems, October 11-15, 2009, pp.1909-1916.

[3] S. M. Kim, J. H. Lee, S. K. Noh, and S. Y. Bang, "Development of the pre-processing algorithm for improving the Optical Flow tracking performance in the ultrasound image," Electronics Journal of the Society, vol. 47, no. 5, pp. 24-32, 2010

[4] B. S. Lee and J. C. Joun, "Tracking method lips of real-time using the optical flow," in Proc. the Korea Internet Information Society Spring Scientific Annual Conference, vol. 8, no. 1, pp. 259-262, 2007.

[5] H. J. Yang and Y. H. Joo, "3-D optical image processing by the BSpline interpolation and template matching," Korea Institute of Intelligent Systems, vol. 19, no. 5, pp. 683-688, 2009.

[6] G. Bradski, A. Kaehler, Learning Opencv: Computer Vision with the Opencv Library, O'REILLY, 2008.

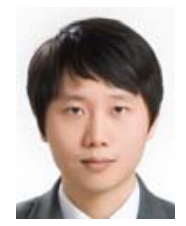

Kyung-Pyo Kim received the B.S degree in Department of Multimedia Communication Engineering from Kyungsung Universty, Pusan, Korea in 2010, where he is currently working toward the joint M.S degree in computer science and engineering in Kyungpook National University. His research interest include computer vision, image processing and pattern recognition.

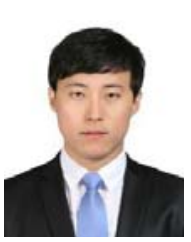

Jae-Hyun Jun received the B.S. degree in Department of Electronic Engineering from Catholic University of Daegu in 2009, and he received his M.S degree in electrical engineering and computer science from Kyungpook National University in 2011. Now he is Ph.D course in School of Computer Science and Engineering in Kyungpook National University.

His research interests include traffic classification, DDoS attack detection, H.264/AVC, H.265/HEVC and Multimedia.

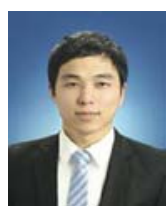

Dong-Sik Park received the B.S degree in Department of Information and Communication Engineering from Kyungsung University, Pusan, Korea, in 2013, Now he is doing M.S course in School of Computer Science and Engineering in Kyungpook National University.

His research interests include human tracking and face tracking.

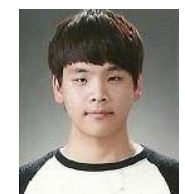

Dong-Joon Lee received the B.S degree from Kyungpook National University, Daegu, Korea, in 2013. Now he is doing M.S course in School of Computer Science and Engineering in Kyungpook National University.

His research interests include face recognition, face detection and pattern recognition.

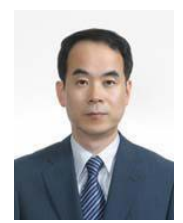

Sung-Ho Kim received the B.S degree in electrical engineering from Kyungpook National University in 1981, and he received his M.S and Ph.D. degree in computer science from Korea Advanced Institute of Science and Technology in 1983 and 1994 respectively. Now he is a professor in Kyungpook National University. 